\title{
Optical properties of polymer nanocomposites
}

\author{
S SRIVASTAVA, M HARIDAS and J K BASU* \\ Department of Physics, Soft Nanomaterials Physics Laboratory, Indian Institute of Science, \\ Bangalore 560 012, India
}

\begin{abstract}
Nanomaterials have emerged as an area of interest motivated by potential applications of these materials in light emitting diodes, solar cells, polarizers, light - stable colour filters, optical sensors, optical data communication and optical data storage. Nanomaterials are of particular interest as they combine the properties of two or more different materials with the possibility of possessing novel mechanical, electronic or chemical behaviour. Understanding and tuning such effects could lead to hybrid devices based on these nanocomposites with improved optical properties. We have prepared polymer nanocomposites of well-defined compositions and studied the optical properties of powders and their thin films. UV-vis absorption spectroscopy on nanocomposite powders and spectroscopic ellipsometry measurements on thin films was used to study the effect of interfacial morphology, interparticle spacing and finite size effects on optical properties of nanocomposites. Systematic shift in the imaginary part of the dielectric function can be seen with variation in size and fraction of the gold nanoparticle. The thickness of the film also plays a significant role in the tunability of the optical spectra.
\end{abstract}

Keywords. Polymer nanocomposites; optical properties; metal nanocomposites.

\section{Introduction}

The characteristic properties of metal nanoparticles and nanocomposites have been the subject of study for a long time because of their unique optical (Nussbaumer et al 2003), thermal, mechanical, electronic and electrical properties (Bohren and Huffman 1983; Kreibig and Vollmer 1995; Abyaneh et al 2006). Plasmon resonance allows for the enhancement and manipulation of local electromagnetic fields at nanoparticle surface due to surface effects and as a result of these singular optical features, noble metal nanoparticles stimulate great interest for implementation in photonics (Maier et al 2003), biotechnology and space applications. Owing to interesting optical properties of nanocomposites which are different from the ones of individual metals, these materials have drawn much attention. Combining metal and polymer together enhances the optical properties of nanometals and also alter the mechanical behaviour of the polymer. The former is of prime importance due to its possible application in polarizers (Lu et al 1997), light stable colour filters (Leon et al 2001), solar cells and optical sensors (Tanaka et al 1991; Tokizaki et al 1991). If the properties of polymer can also be tuned in correct direction with enhanced optical properties of metals, these materials could be best candidates for space application. The size dependent structural, optical, thermal and electronic

\footnotetext{
*Author for correspondence (basu@physics.iisc.ernet.in)
}

properties can be tailored to be suitable for device applications by varying the particle size. Small changes of the configuration of the composite concerning the metal fraction as well as the size and shape of the nanoparticles can lead to dramatic changes in the electrical and optical properties. In the last few decades, the study of optical properties of nanocomposites has been stimulated by promising applications. Significant changes in thermal (Srivastava and Basu 2007a) as well as optical properties (Haridas et al 2008) can be observed in properties of such materials even for very small fraction of the nanoparticles. However, as attractive such nanocomposites may be, the process of blending or dispersing nanoparticles in a polymer matrix has proven to be problematic. Once well dispersed in a matrix, the intrinsic properties of a nanostructured material are determined by its size, shape, composition, crystallinity and structure. Polymer system of the noble metals, copper, gold and silver, show very intense colours, which are absent for the bulk materials as well as for the individual atoms. Their origin is attributed to the surface plasmon resonance (SPR), collective oscillation of free conduction electrons induced by an interacting electromagnetic field. SPR was first quantitatively described by Mie by solving Maxwell's equation with the appropriate boundary conditions for spherical particles. The total extinction cross-section composed of absorption and scattering is given as a summation over all electric and magnetic multipole oscillations. Mie's (1908) theory is successful in reproducing the extinction spectra from gold nanoparticles for size $>20 \mathrm{~nm}$. However, as size decreases the additional effects come in and plasmon 
peak shifts towards lower wavelength which can be explained by introducing size dependent dielectric constant in Mie theory.

The aim of this work is to study the optical properties of gold nanocomposites by changing the size and fraction of gold nanoparticles. For these experiments, we have prepared well dispersed, stable and homogeneous gold nanoparticles in the polymer matrix. The size of the particles was varied over wide range by changing the concentration and molecular weight of the capping agent. The optical properties of nanocomposite films of various thicknesses with different sizes and volume fraction of gold nanoparticles has been studied using spectroscopic ellipsometry.

\section{Experimental}

\subsection{Synthesis and materials}

The gold nanocomposites were synthesized (Srivastava and Basu 2007b) by reduction of $\mathrm{HAuCl}_{4}$ with $\mathrm{NaBH}_{4}$ in the presence of PMMA. When the reduction of gold chloride was carried out by $\mathrm{NaBH}_{4}$, the colour of the solution changed from yellow to brownish red indicating the formation of gold nanoparticles (figure 1). All chemicals, hydrogen tetrachloro auric acid $\left(\mathrm{HAuCl}_{4}\right)$, PMMA (molecular weights, $120 \mathrm{k}$ ), sodium borohydride $\left(\mathrm{NaBH}_{4}\right)$ were obtained from Sigma-Aldrich Chemicals and were used without any treatment. Ethanol was obtained from Merck. Deionized water (18.2 mega ohm, Barnstead) was used in all experiments. The thin film samples were made by spin coating nanocomposite solution on silicon substrate at different speeds.

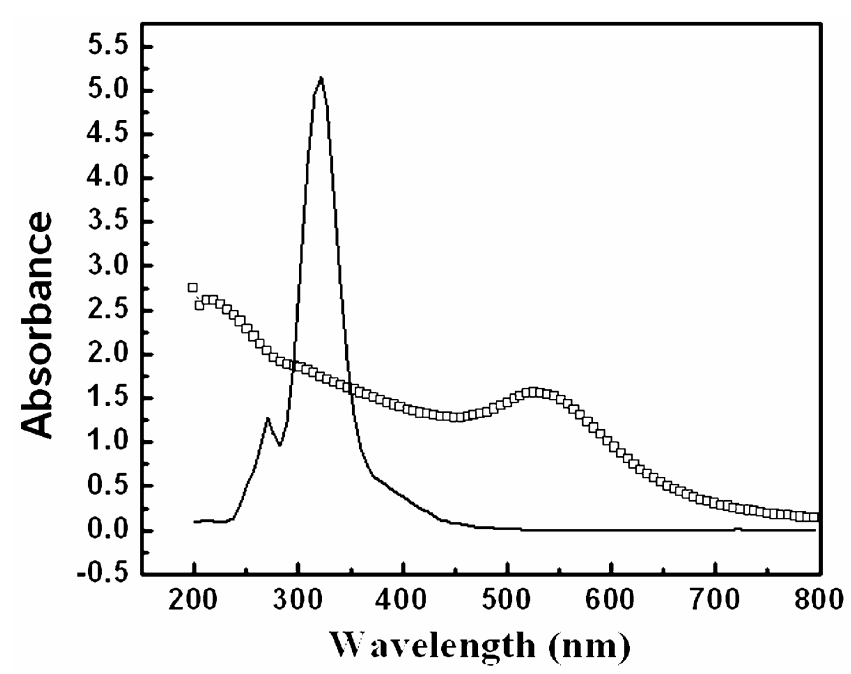

Figure 1. UV-vis absorption spectroscopy of gold nanoparticles in ethanol-water solution. Strong absorption at $520 \mathrm{~nm}$ for nanoparticle sample is clear signature for the formation of gold nanoparticles. Solid line represents the absorption for $\mathrm{HAuCl}_{4}$ solution.

\section{Characterization}

UV-vis spectra of the polymer nanocomposites were taken using GBC Cintra $40 \mathrm{UV}$-vis spectrometer (range 200-800 nm). Figure 1 shows the surface plasmon absorption for gold nanoparticles confirming the formation of gold nanoparticles. The peak assigned to $\mathrm{HAuCl}_{4}$ in the absorption spectra at $300 \mathrm{~nm}$ due to bulk plasmon disappears and can be seen at $520 \mathrm{~nm}$ for gold nanoparticles due to surface plasmon resonance.

Bright field images were obtained using Tecnai F 30 High Resolution Transmission Electron Microscope (TEM). Figure 2 shows the TEM images of well dispersed gold nanoparticles of different sizes in the PMMA matrix. Small concentration of capping agent results in particles of large size but the effective distribution of particle size also becomes broader. Stable gold particles
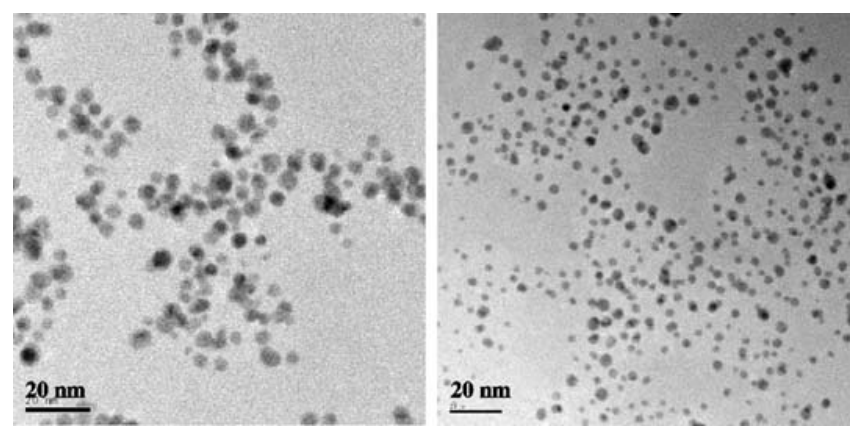

Figure 2. TEM images of gold nanoparticles of mean sizes, $4.5 \mathrm{~nm}$ (left) and $3.5 \mathrm{~nm}$ (right), in PMMA matrix showing their relatively homogeneous dispersion.

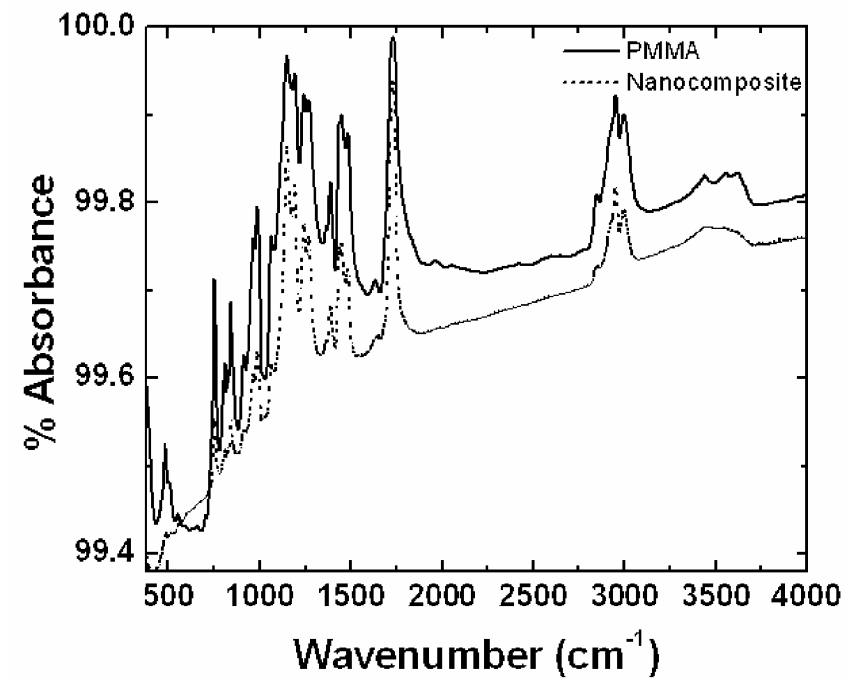

Figure 3. FTIR spectra of neat PMMA (solid line) and PMMA embedded with $6 \mathrm{~nm}$ diameter gold nanoparticle (dashed line). No chemical shift has been observed which indicates the absence of any chemical attachment of the polymer chain at the particle-polymer interface. 
of various sizes in the range $2 \cdot 5-10 \mathrm{~nm}$ were obtained by tuning the concentration and also the molecular weight of PMMA.

Fourier transform infrared spectroscopy (FTIR, Bruker IFS $66 \mathrm{~V} / \mathrm{S}$ ) was used to study the chemical affinity of PMMA segments towards the surface of these nanoparticles. Figure 3 shows that the FTIR spectra of neat PMMA and PMMA capped gold nanoparticles are almost identical with no noticeable shifts in the positions of the lines, indicating a non-wetting polymer-particle interfacial

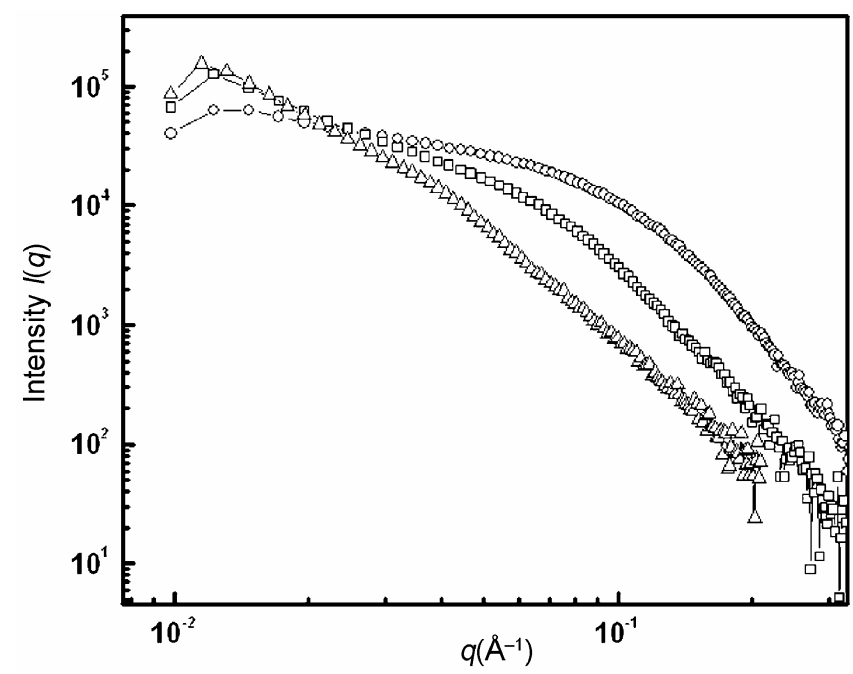

Figure 4. SAXS data in full $q$-range for PMMA embedded with various sizes in increasing order $\left(O_{-}-\triangle-\triangle\right)$ of gold nanoparticles. The scattering profiles for all samples show no indication of aggregation or clustering effects in nanocomposites.

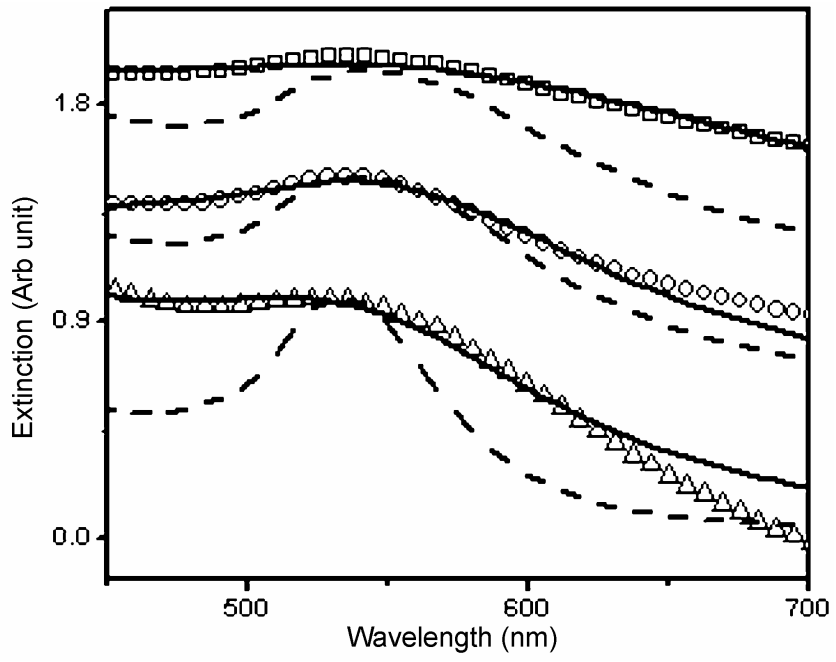

Figure 5. UV-vis absorption spectra on polymer nanocomposite for particles of sizes $4(\square), 2(O)$ and $5(\triangle)$ (in nm). Solid line indicates the fitted curve using size dependent dielectric function taking into account PMMA capping thickness and dashed line represents the calculated curve based on Mie theory, using bulk dielectric constant. surface. Small angle system with X-ray scattering (SAXS) system was used to obtain information about the interfacial morphology as has been described earlier (Srivastava et al 2007a).

Spectroscopic ellipsometry (Sentech SE 850) was used to extract the optical constants for thin films of nanocomposite with different fractions and sizes of the nanoparti-

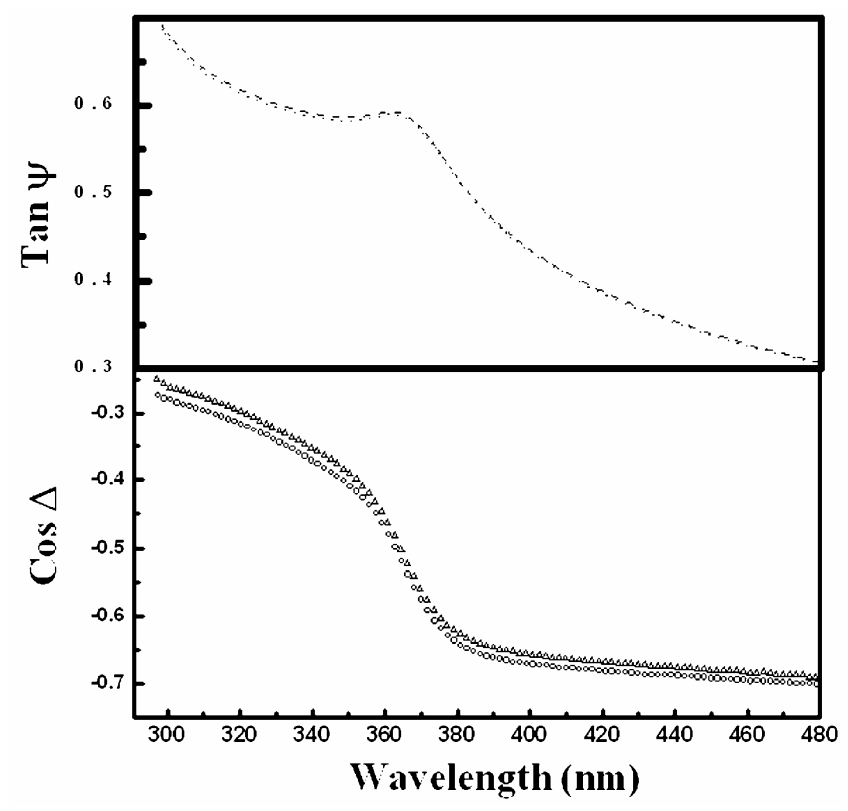

Figure 6. Ellipsometry data on nanocomposite films of thickness, $15 \mathrm{~nm}$, with gold nanoparticles of size, $6 \mathrm{~nm}$ and different volume fractions (Top panel: $\tan \psi$ for volume fractions, $1.2 \%$ (dash) and $0.6 \%$ (dot); bottom panel: $\cos \Delta$ for volume fractions, $1 \cdot 2 \%(\triangle)$ and $0.6 \%(O)$.

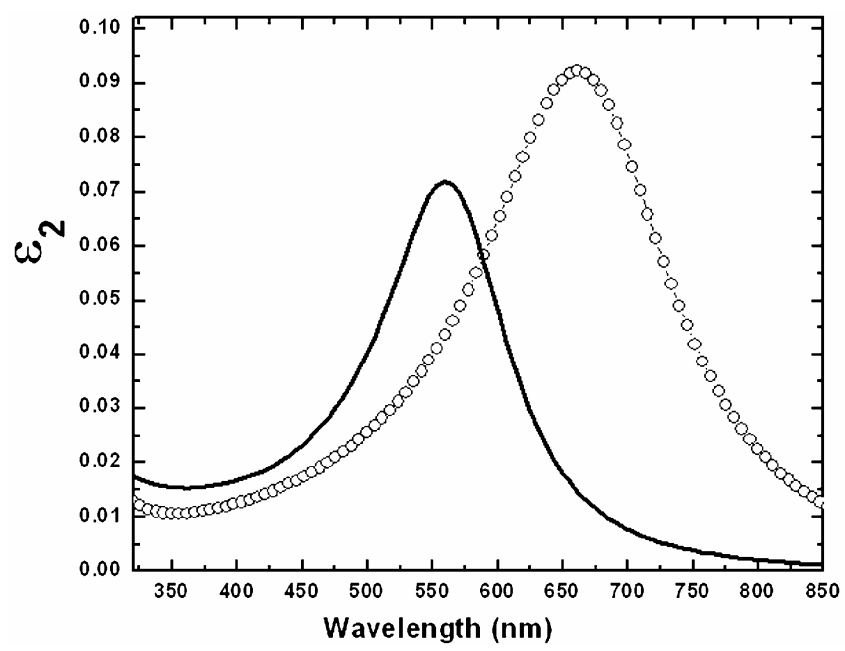

Figure 7. Imaginary part of dielectric function $\left(\varepsilon_{2}\right)$ of gold nanoparticles in nanocomposite films with thickness, $15 \mathrm{~nm}$. The effect of volume fraction $(1.2 \%(0)$ and $0.6 \%(-))$ of gold nanoparticles of size, $6 \mathrm{~nm}$, in the composite, on the effective $\varepsilon_{2}$ of gold nanoparticles is clearly shown. 
cle. The optical constant as a function of wavelength can be extracted at variable angles. All measurements to study the effect of confinement of nanoparticles on their optical properties of thin films were done at $70^{\circ}$. We prepared thin films of various thicknesses in the range 15$30 \mathrm{~nm}$ using the PMMA-gold nanocomposite on silicon wafers by spin coating method. Films with homogeneous surfaces were obtained for reasonably small thickness, for films of larger thickness the surface becomes rough due to aggregation of particles. We have tried making films on several types of substrate glass, quartz and silicon and after considerable experimentation found that silicon wafers are the best choice and gives good quality films. At higher concentration of the fillers the particles come together and form clusters which adds to the difficulty of getting smooth films of larger thickness. Thus all ellipsometry measurements were done on films of variable thickness and the optical properties were extracted for different sizes and composition of nanoparticles.

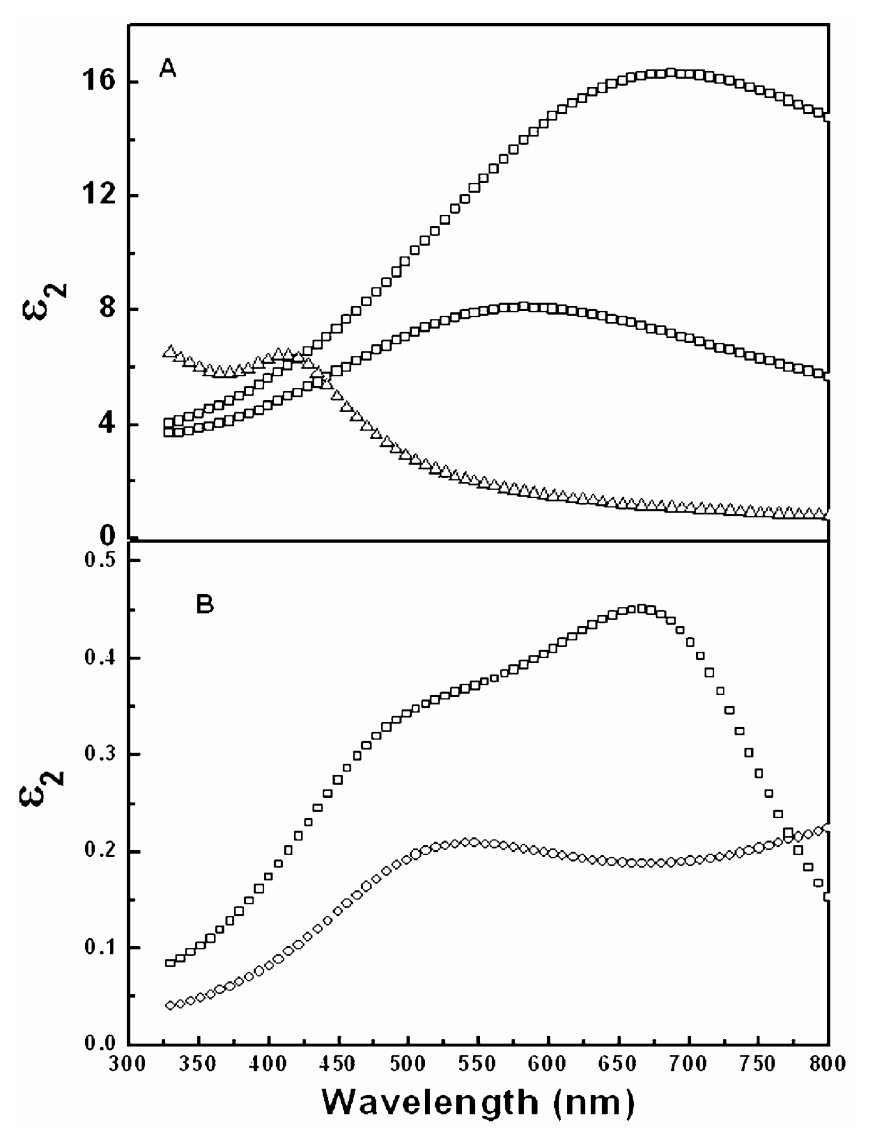

Figure 8. Imaginary part of dielectric function $\left(\varepsilon_{2}\right)$ for nanocomposite films with variable thickness but fixed size $(6 \mathrm{~nm})$ and volume fraction $(6.6 \%)$ of the gold nanoparticles. A. Obtained $\varepsilon_{2}$ values for gold particles in the nanocomposite films with thicknesses, $30 \mathrm{~nm}(O)$ and $25 \mathrm{~nm}(\square)$. Red shift in $\varepsilon_{2}$ can be observed with respect to bulk gold $(\triangle)$ and $\mathbf{B}$. comparison of the $\varepsilon_{2}$ values for the total nanocomposite layers (PMMA and gold) for the same films as in (A).

\section{Results and discussion}

Our results for different concentrations of polymer showed that fairly monodisperse and small size particles can be obtained for large concentration of polymer. Formation of the nanoparticle was confirmed by the UV-vis spectra, showing strong absorption at $520 \mathrm{~nm}$ due to surface plasmon. The sizes of the nanoparticle were characterized by TEM and SAXS. The estimation of size from the SAXS data is in good agreement with the TEM data. Figure 4 shows the SAXS spectrum for nanoparticles of different sizes. It can be clearly seen from the data that with increase in the size of the nanoparticle the onset of Porod region shifts towards lower value of wave vector, $q$.

We have also studied the optical absorption spectra of several gold-PMMA nanocomposite powders (figure 5) and observed that the absorption calculated based on Mie theory using bulk dielectric constant (Johnson and Christy 1972) alone, cannot account for the observed shift and width of plasmon peak. However, reasonably good match was obtained by fitting the dielectric constant using recently proposed analytical model (Etchegoin et al 2006; Haridas et al 2008). We have also taken into account the PMMA capping thickness as obtained from SAXS data. This points to the importance of the capping layer on the optical properties of nanoparticles embedded in polymers. Figure 6 shows the $\tan \psi$ and $\cos \Delta$ spectra obtained from ellipsometry measurement on polymer nanocomposite thin films containing gold nanoparticles of various fractions in the PMMA matrix.

The data were analysed using well known MaxwellGarnet effective medium approximation (Bohren and Huffman 1983) to model the nanocomposite layer to extract the optical constant. Using this model we were also able to, independently, extract the volume fraction of the gold nanoparticles in the matrix as well as the optical constants for individual components of the nanocomposite layer. Figure 7 represents the imaginary part of the dielectric constants extracted from the fits to the data in figure 6 . We observed significant red shift in the peak position in the imaginary part of the dielectric function $\left(\varepsilon_{2}\right)$ with increase in the volume fraction of gold nanoparticles. In figure $8(\mathrm{~A})$, the imaginary part of dielectric function for two different film thicknesses have been compared with the bulk values. Significant red shift with respect to bulk value can be observed with increase in thickness of the nanocomposite films.

Figure 8(B) shows the thickness dependence of the imaginary part of dielectric function for gold nanoparticles of size, $6 \mathrm{~nm}$ and volume fraction, $6 \cdot 6 \%$. By decreasing the thickness of the films we were able to shift the absorption towards high wavelength for same size of gold nanoparticle.

For nanocomposite layer of $25 \mathrm{~nm}$ thickness, two broad absorptions can be observed. This gives us an additional parameter to tune the optical properties of the composite 
films. The red-shift in the absorption maxima with increasing fraction as well as increased absorption is clearly evident. This is very useful since this indicates the ability to obtain optical coatings with tunable optical properties.

\section{Conclusions}

Systematic variation of the absorption peak width and position as a function of fraction of gold nanoparticles in PMMA was obtained. This is very useful since this indicates the ability to obtain optical coatings with tunable optical properties. We have also found some very interesting behaviour of the optical absorption as a function of interfacial morphology. This gives us an additional parameter to tune the optical properties of the composites. This work demonstrates possibility of creating stable polymer nanocomposite powders and films with interesting optical and electronic properties.

\section{Acknowledgements}

We would like to acknowledge the ISRO-IISc, Space Technology Cell, for providing financial assistance for this work. We thank DST-IISc Nanoscience Initiative for use of TEM facility.

\section{References}

Abyaneh M K, Pasricha R, Gosavi S W and Kulkarni S K 2006 Nanotechnol. 174129

Bohren C F and Huffman D F 1983 Absorption and scattering of light by small particles (New York: John Wiley \& Sons)

Etchegoin P G, Le Ru E C and Mayer M 2006 J. Chem. Phys. 25164705

Haridas M, Srivastava S and Basu J K 2008 EPJD (submitted) Inouye H, Tanaka K, Tanahashi I and Hirao K 1998 Jpn J. Appl. Phys. 37 L1520

Johnson P B and Christy R W 1972 Phys. Rev. B6 4370

Kreibig U and Vollmer M 1995 Optical properties of metal clusters (Springer: Berlin)

Leon A G, Dirix Y, Staedler Y, Feldman K, Hhner G, Caseri W R and Smith P 2001 Appl. Opt. 394847

Lu A H, Lu G H, Kessinger A M and Foss C A 1997 J. Phys. Chem. B101 9139

Maier S A et al 2003 Nat. Mater. 2229

Mie G 1908 Phys. Lpz 25377

Nussbaumer R J, Caseri W R, Smith P and Tervoort T 2003 Macromol. Mater. \& Eng. 28844

Srivastava S and Basu J K 2007a Phys. Rev. Lett. 98165701

Srivastava S and Basu J K 2007b J. Nano. Sci. Technol. 7 2101

Tokizaki T, Nakamura A, Kaneko S, Uchida K, Omi S, Tanji H and Asahara Y 1991 Appl. Phys. Lett. 65941 